\title{
Complexity measure and quantum shape-phase transitions in the two-dimensional limit of the vibron model
}

\author{
E. Romera ${ }^{1,2}$, M. Calixto ${ }^{3}$ and Á. Nagy ${ }^{4}$ \\ 1 Instituto Carlos I de Física Teórica y Computacional, \\ Universidad de Granada, Fuentenueva s/n, 18071 Granada, Spain \\ 2 Departamento de Física Atómica, \\ Molecular y Nuclear, Universidad de Granada, \\ Fuentenueva s/n, 18071 Granada, Spain \\ ${ }^{3}$ Departamento de Matemática Aplicada, Universidad de Granada, \\ Fuentenueva $s / n, 18071$ Granada, Spain and \\ 4 Department of Theoretical Physics, \\ University of Debrecen, H-4010 Debrecen, Hungary
}

(Dated: March 21, 2014)

\begin{abstract}
We obtain a characterization of quantum shape-phase transitions in the terms of complexity measures in the two-dimensional limit of the vibron model based on the spectrum generating algebra $U(3)$. Complexity measures (in terms of the Rényi entropies) have been calculated for different values of the control parameter for the ground state of this model giving sharp signatures of the quantum shape-phase transition from linear to bent molecules.
\end{abstract}




\section{INTRODUCTION}

Quantum phase transitions (QPT) are a fundamental part of quantum many body theory and can be considered as an extension of classical phase transitions to zero absolute temperature. QPTs result from the variation of quantum fluctuations. Generally speaking, one finds different quantum phases connected to specific geometric configurations of the ground state and related to distinct dynamic symmetries of the Hamiltonian. The QPT occurs as a function of a control parameter $\xi$ that appears in the Hamiltonian $H$. For us it will appear in the form of a convex combination $H(\xi)=(1-\xi) H_{1}+\xi H_{2}$. At $\xi=0$ the system is in phase I, characterized by the dynamical symmetry $G_{1}$ of $H_{1}$, and at $\xi=1$

the system is in phase II, characterized by the dynamical symmetry $G_{2}$ of $H_{2}$. At some critical point $\xi_{c} \in(0,1)$ there is an abrupt change in the symmetry and structure of the ground state wavefunction. This is the case of the so-called 'vibron models' (see e.g. [1-6]), interacting boson models which exhibits a second order shape-phase transition from linear to bent. These models have been used to study the rovibrational properties in diatomic and polyatomic molecules. The vibron model was introduced by Iachello in the 80's for the description in an algebraic manner of molecular structure (see standard text books [8, 9] on the subject). It is based on the spectrum generating algebra $U(4)$ and it includes a full description of rovibrational degrees of freedom for a diatomic molecule. The description of polyatomic molecular species is achieved through the coupling of several $U(4)$ algebras. Two limits of this model were later introduced, to avoid the mathematical complexities of the full rovibrational description, the one-dimensional limit -based on the $U(2)$ dynamical algebra- and the two dimensional limit -based on the $U(3)$ dynamical algebra- which is the simplest model retaining angular momentum quantum numbers. We chose the latter model to perform calculations of the statistical complexity accross the shape-phase transition from linear to bent, two configurations that appear in triatomic molecules. The importance of shape QTP's in molecular modeling relies on the fact that molecular species exist with a nonrigid structure, intermediate between rigid linear and bent, and a unified description of all configurations within the framework of a single approach (as the vibron model does) is very challenging, since most common approaches so far have concentrated on linear or quasi-linear geometries.

On the other hand, information entropies and statistical complexities have a role of 
growing importance in describing quantum phenomena. Different simple atomic systems [10-16] were studied with Fisher [17] and Shannon [18] information [19-21]. These systems display remarkable properties when the so called LMC complexity $[22,23]$ is computed on them [16, 24]. Recently, it has been shown that Shannon and Rényi entropies [25-27] Wehrl entropies [28, 29], entanglement entropies [30] and Rényi-Fisher products [31] detect QPTs in several atomic and chemical quantum models. In this article we show that the generalized complexity mesures [32] are also excellent descriptors of QPT in the vibron model for molecules.

Complexity is a general indicator of structure and correlation. Therefore, it is useful in chemistry or in molecular modeling. We mention by passing that the special case of LMC complexity, (that is, $\alpha=1$ and $\beta=2$ ) has proved to be useful in studying several chemical and physical properties. In Reference [33] it was found that molecules with low number of electrons possess low complexities, while larger number of electrons possess larger complexities. Studying hardness and ionization potentials, they concluded that molecules that are more stable chemically, possess low complexity values.

This article is organized as follows: in section II we remind the reader the two-dimansional limit of the vibron model, in section III we study the description of the QPT in this model by means of a complexity measure. Finally some conclusions are given.

\section{U(3) VIBRON MODEL AND SHAPE QPT}

The two dimensional vibron model, based in the $U(3)$ symmetry spectrum generating algebra, describes a planar molecular system containing a dipole degree of freedom [1]. It is an appropriate model for the study of critical properties because it is the simplest model which still retains the basics of more complicated three-dimensional models and embodies the necessary physical ingredients to reproduce the spectroscopic signatures of rigidly-linear, rigidly-bent and floppy molecular configurations $[5,6]$. The basic physical realization of such a system is the bending vibrational mode of a molecule $[2,3]$. In the $U(3)$ vibron model one finds different shape phases connected to specific geometric configurations of the ground state and related to distinct dynamic symmetries of the Hamiltonian [4]. Let us give a brief of the basic ingredients to construct this Hamiltonian. In this model the elementary excitations are described by creation and annihilation two-dimensional vector 
$\tau$-bosons $\left\{\tau_{x}^{\dagger}, \tau_{y}^{\dagger}, \tau_{x}, \tau_{y}\right\}$ and a scalar $\sigma$-boson $\left\{\sigma^{\dagger}, \sigma\right\}$. It is convenient to introduce circular bosons: $\tau_{ \pm}=\mp\left(\tau_{x} \mp i \tau_{y}\right) / \sqrt{2}$. The essential Hamiltonian of this system is written in terms of bilinear products of these creation and annihilation operators, which lead to up to nine generators of the corresponding $U(3)$ algebra. Concretely, the essential Hamiltonian [4] is only written in terms of the following five generators:

$$
\begin{gathered}
\hat{n}=\tau_{+}^{\dagger} \tau_{+}+\tau_{-}^{\dagger} \tau_{-}, \hat{n}_{s}=\sigma^{\dagger} \sigma \\
\hat{l}=\tau_{+}^{\dagger} \tau_{+}-\tau_{-}^{\dagger} \tau_{-}, \\
\hat{D}_{+}=\sqrt{2}\left(\tau_{+}^{\dagger} \sigma-\sigma^{\dagger} \tau_{-}\right), \hat{D}_{-}=\sqrt{2}\left(-\tau_{-}^{\dagger} \sigma+\sigma^{\dagger} \tau_{+}\right),
\end{gathered}
$$

where $\hat{n}$ and $\hat{n}_{s}$ are number operator of vector and scalar bosons, respectively, $\hat{l}$ is the twodimensional angular momentum and $\hat{D}_{ \pm}$is the dipole operator. We have to take into account that the total number of bosons $\hat{N}=\hat{n}+\hat{n}_{\sigma}$ and the two-dimensional angular momentum $\hat{l}$ are conserved. With these ingredients, the essential Hamiltonian is given by

$$
\hat{H}=(1-\xi) \hat{n}+\xi \frac{N(N+1)-\hat{W}^{2}}{N-1},
$$

where $N$ is the "size" of the system [it labels the totally symmetric $(N+1)(N+2) / 2$ dimensional representation $[N]$ of $U(3)]$, the operator $\hat{W}^{2}=\left(\hat{D}_{+} \hat{D}_{-}+\hat{D}_{-} \hat{D}_{+}\right) / 2+\hat{l}^{2}$ is the squared angular momentum of the $S O(3)$ subalgebra, and $\xi \in[0,1]$ is a control parameter. In the thermodynamic (mean field or large size) limit, where number of bound states becomes large, $N \rightarrow \infty$, there is a shape QPT at a critical value $\xi_{c}=0.2$ of the control parameter $\xi$, which marks the boundary between the two geometrical phases: the linear (for $\xi<\xi_{c}$ ) and the bent (for $\xi>\xi_{c}$ ) phases (see later on this section for a more precise explanation).

We will consider the following state space basis in order to solve the eigenvalue problem

$$
|N ; n, l\rangle=\frac{\left(\sigma^{\dagger}\right)^{N-n}\left(\tau_{+}^{\dagger}\right)^{\frac{n+l}{2}}\left(\tau_{-}^{\dagger}\right)^{\frac{n-l}{2}}}{\sqrt{(N-n) !\left(\frac{n+l}{2}\right) !\left(\frac{n-l}{2}\right) !}}|0\rangle,
$$

where the bending quantum number $n=N, N-1, N-2, \ldots, 0$ and the angular momentum $l= \pm n, \pm(n-2), \ldots, \pm 1$ or $0(n=$ odd or even $)$ are the eigenvalues of $\hat{n}$ and $\hat{l}$, respectively. The matrix elements of $\hat{W}^{2}$ can be easily derived (see e.g. [4]):

$$
\begin{aligned}
& \left\langle N ; n^{\prime}, l\left|\hat{W}^{2}\right| N ; n, l\right\rangle= \\
& {\left[(N-n)(n+2)+(N-n+1) n+l^{2}\right] \delta_{n^{\prime}, n}} \\
& -[(N-n+2)(N-n+1)(n+l)(n-l)]^{\frac{1}{2}} \delta_{n^{\prime}, n-2} \\
& -[(N-n)(N-n-1)(n+l+2)(n-l+2)]^{\frac{1}{2}} \delta_{n^{\prime}, n+2} .
\end{aligned}
$$


The ground state energy in the thermodynamic limit $N \rightarrow \infty$ is recovered by using ground state ansätze in terms of (semi-classical) coherent states

$$
|N ; r\rangle \equiv \frac{1}{\sqrt{N !}}\left(b_{c}^{\dagger}\right)^{N}|0\rangle, \quad b_{c}^{\dagger}=\frac{1}{\sqrt{1+r^{2}}}\left(\sigma^{\dagger}+r \tau_{x}^{\dagger}\right)
$$

with $r$ a free variational real parameter and $b_{c}^{\dagger}$ the boson condensate. Other rotationally equivalent possibilities can also be also considered [7]. The variational parameter $r$ is fixed by minimizing the ground state energy functional 'per particle' (see e.g. [4]):

$$
\begin{aligned}
\mathcal{E}_{\xi}(r) & =\frac{\langle N ; r|\hat{H}| N ; r\rangle}{N}=(1-\xi) \frac{\langle N ; r|\hat{n}| N ; r\rangle}{N}+\xi \frac{N(N+1)-\left\langle N ; r\left|\hat{W}^{2}\right| N ; r\right\rangle}{N(N-1)} \\
& =(1-\xi) \frac{r^{2}}{1+r^{2}}+\xi\left(\frac{1-r^{2}}{1+r^{2}}\right)^{2} .
\end{aligned}
$$

From $\partial \mathcal{E}_{\xi}(r) / \partial r=0$ one gets the 'equilibrium radius' $r_{e}$ and the ground state energy $\mathcal{E}_{\xi}$ as a function of the control parameter $\xi$ :

$$
\begin{aligned}
& r_{e}(\xi)= \begin{cases}0, & \xi \leq \xi_{c}=1 / 5, \\
\sqrt{\frac{5 \xi-1}{3 \xi+1}}, & \xi>\xi_{c}=1 / 5\end{cases} \\
& \mathcal{E}_{\xi}\left[r_{e}(\xi)\right]= \begin{cases}\xi, & \xi \leq \xi_{c}=1 / 5 \\
\frac{-9 \xi^{2}+10 \xi-1}{16 \xi}, & \xi>\xi_{c}=1 / 5 .\end{cases}
\end{aligned}
$$

Then one finds that $d^{2} \mathcal{E}_{\xi}\left[r_{e}(\xi)\right] / d \xi^{2}$ is discontinuous at $\xi_{c}=1 / 5$ and the shape phase transition, from linear $(\xi \leq 1 / 5)$ to bent $(\xi>1 / 5)$ is said to be of second order. For triatomic molecules, the (dimensionless) algebraic variational coordinate $r$ has been related to a physical angular displacement $[2,4,5]$ (or 'bending angle') $\theta \sim r / a$, with $a$ the equilibrium bond length, which reflects the degree of distortion of the molecular framework from linearity $(r=0)$.

For a finite number $N$ of bound states, a numerical diagonalization of the Hamiltonian (2) must be done for each value of the control parameter $\xi$. The ground state wave function is given

$$
\left|\psi_{\xi}^{(N)}\right\rangle=\sum_{n=0}^{N} \sum_{m=0}^{n} c_{n, m}^{(N)}(\xi)|N ; n, l=n-2 m\rangle,
$$

where the coefficients $c_{n, m}^{(N)}(\xi)$ are obtained by diagonalization of the Hamiltonian (2) in terms of the basis vectors (3). In order to calculate complexity measures for the ground state density function $f=|\psi|^{2}$, we must introduce position (and momentum) representations. 
Denoting by $\left(a_{1}, a_{2}, a_{3}\right) \equiv\left(\sigma, \tau_{+}, \tau_{-}\right)$the three oscillator operators, position and momentum operators are defined in the standard way as $\hat{x}_{i}=\frac{1}{\sqrt{2}}\left(a_{i}^{\dagger}+a_{i}\right)$ and $\hat{p}_{i}=\frac{i}{\sqrt{2}}\left(a_{i}^{\dagger}-a_{i}\right)$, respectively, for $i=1,2,3$. We should say that the "position" $\mathbf{r}=\left(x_{1}, x_{2}, x_{3}\right)$ is not directly related with the true space coordinates of the molecule, but it provides a useful representation to write the wave packet. The basis functions (3) are then written in position representation

$$
\begin{aligned}
\phi_{n, l}^{N}(\mathbf{r})=\langle\mathbf{r} \mid N ; n, l\rangle= & \frac{2^{-N / 2} \pi^{-3 / 4} e^{-\mathbf{r}^{2} / 2}}{\sqrt{(N-n) !\left(\frac{n+l}{2}\right) !\left(\frac{n-l}{2}\right) !}} \\
& \times H_{N-n}\left(x_{1}\right) H_{\frac{n+l}{2}}\left(x_{2}\right) H_{\frac{n-l}{2}}\left(x_{3}\right) .
\end{aligned}
$$

in terms of Hermite polynomials $H_{n}(x)$. In this model, wave functions in position and momentum representation are the same. This is easy to check taking into account the properties of the Fourier transform of the $e^{-x^{2}} H_{n}(x)$ and the fact that the system is parity invariant. As position and momentum wave functions are the same in this particular model, in the following we will only consider position space expressions. We will write $\psi_{\xi}^{(N)}(\mathbf{r})=$ $\left\langle\mathbf{r} \mid \psi_{\xi}^{(N)}\right\rangle$ the corresponding wave function in position representation and $f_{\xi}^{(N)}(\mathbf{r})=\left|\psi_{\xi}^{(N)}(\mathbf{r})\right|^{2}$ (or simply $f(\mathbf{r})$ when there is no confusion) the ground state position density distribution.

\section{COMPLEXITY AND DESCRIPTION OF THE QPT IN THE VIBRON MODEL}

Consider a $D$-dimensional nonnegative function $f(\mathbf{r})$. A complexity measure of $f$ can be defined as [32]:

$$
C_{f}^{(\alpha, \beta)} \equiv e^{R_{f}^{(\alpha)}-R_{f}^{(\beta)}}, \quad 0<\alpha, \beta<\infty,
$$

where $R_{f}^{(\alpha)}$ is the Rényi entropy of the function $f$ given by

$$
R_{f}^{(\alpha)}=\frac{1}{1-\alpha} \ln \int f^{\alpha}(\mathbf{r}) d \mathbf{r} \text { with } 0<\alpha<\infty \text { and } \alpha \neq 1
$$

and $R_{f}^{(1)}=S_{f}=-\int f(\mathbf{r}) \ln f(\mathbf{r}) d \mathbf{r}$ with $S_{f}$ the so-called Shannon entropy of $f$ [18]. That is, the Rényi entropy reduces to the Shannon entropy if $\alpha \rightarrow 1$. Eq. (9) is the generalization of the so called LMC complexity measure [23]:

$$
C_{L M C}=H_{f} D_{f},
$$

$H_{f}=e^{S_{f}}$ is the Shannon entropy power, while $D_{f}=\int f^{2}(\mathbf{r}) d \mathbf{r}=e^{-R_{f}^{(\beta=2)}}$ is the disequilibrium. A one-parameter extension of the LMC complexity measure [34] is given by the 
selection $\beta=2$ in Eq. (9). The most general form of this kind of family of complexities can be defined by Eq. (9). This definition leads to several appealing features. The complexity measure (9) verifies the mathematical properties of inversion symmetry, monotonicity, invariance under translations, rescaling transformations and replication, and near continuity (For an extensive description see [32]). The generalized complexity is an increasing function of $\beta$ for a fixed $\alpha$. That means that increasing the value of $\beta$ we can 'enlarge' the changes in the complexity. That is the advantage of examining complexity instead of the Renyi entropy. In studying the QPT it can be useful to magnify the vicinity of the transition point. It can especially be helpful if we want to apply the generalized complexity as a control parameter.

Figure 1 shows the complexity measure for the ground state density as a function of $\xi$ for $(\alpha, \beta)=(1,3 / 2)$ (left) and $(3 / 4,3 / 2)$ (right), for $N=8,16$ and 20 . The density has been computed numerically. The complexity measure is quasi-constant in the linear phase and is

a decreasing function for $\xi \geq 0.2$. That is, $C_{f}^{(\alpha, \beta)}$ is quasi-constant for linear and quasi-linear molecules and a decreasing function of $\xi$ for bent molecules So the complexity measure is a detector of the quantum phase transition and could be considered as an order parameter in the system to distinguish different type of molecules. We would like to point out that we have considered other values of the parameters $\alpha$ and $\beta$ and the results are analogous.

\section{CONCLUSIONS}

In this work, a complexity measure has been proposed to describe a QPT in the $U(3)$ vibron model for molecules. We have shown that this information measure has different behavior for each phase in this model: (i) it is quasi-constant in the linear phase and (ii) it is a decreasing function of the control parameter in the model in the bent phase. This analysis can be extended to other systems where QPTs occur to prove the general validity of the results.

\section{Acknowledgments}

This work was supported by the Projects: FIS2011-24149 and FIS2011-29813-C02-01 (Spanish MICINN), PP2012-PI04 (University of Granada). The work is also supported by the TAMOP 4.2.2.A-11/1/KONV-2012-0036 project. The project is co-financed by the Eu- 

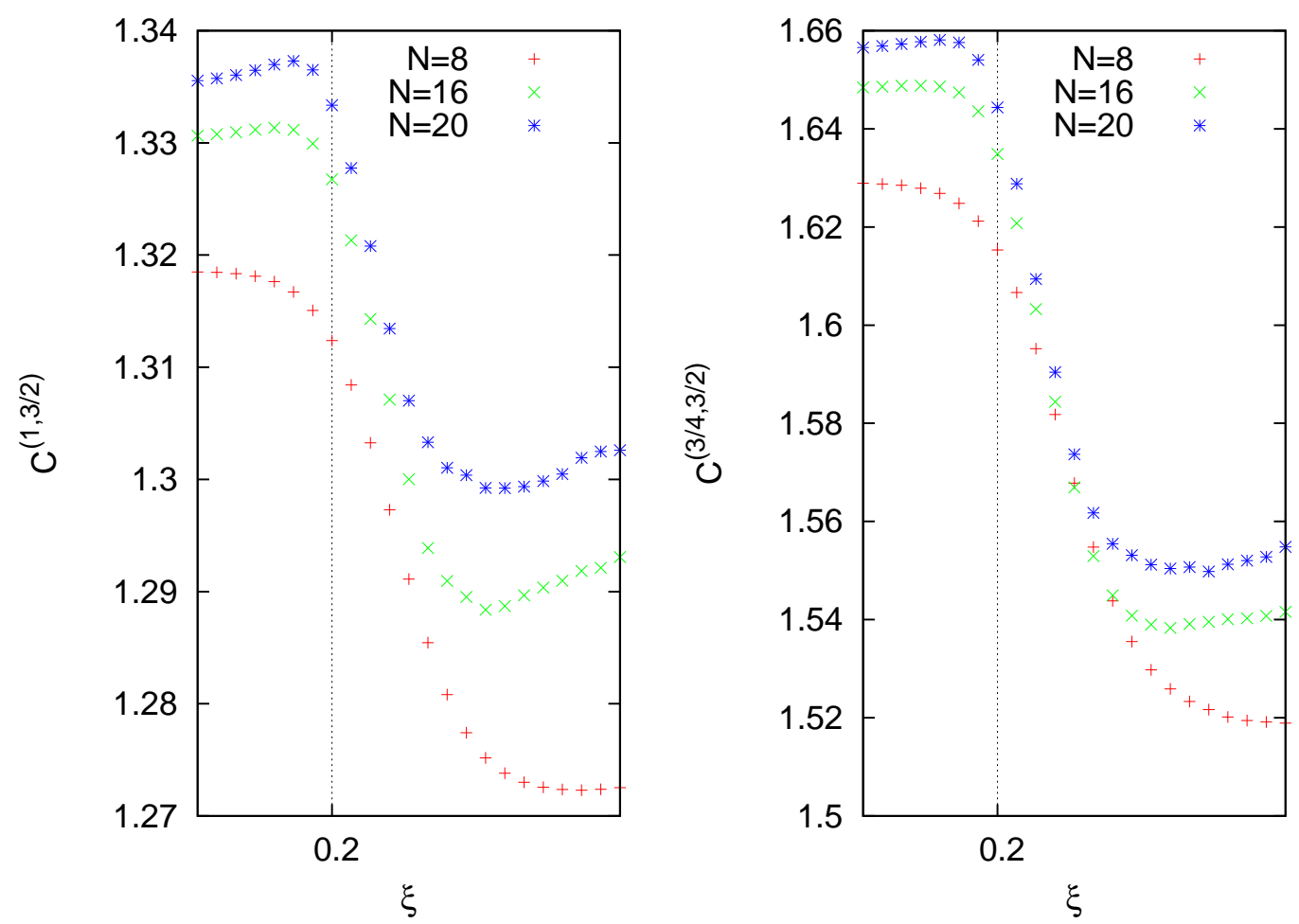

FIG. 1: (Color online) Complexity measure $C_{f_{\xi}}^{(\alpha, \beta)}$ for the exact (numerical) ground state of the vibron model as a function of $\xi$ for $(\alpha, \beta)$ (left) $(1,3 / 2)$ and (right) $(3 / 4,3 / 2)$ for $N=8,16$ and 20.

ropean Union and the European Social Fund. Grant OTKA No. K 100590 is also gratefully acknowledged.

[1] F. Iachello and S. Oss. J. Chem. Phys., 104, 6956 (1996).

[2] F. Iachello, F. Pérez-Bernal, P.H. Vaccaro, Chem. Phys. Lett. 375, 309 (2003).

[3] F. Pérez-Bernal, L. F. Santos, P. H. Vaccaro, and F. Iachello. Chem. Phys. Lett. 414, 398 (2005).

[4] F. Pérez-Bernal, F. Iachello, Phys. Rev. A 77, 032115 (2008).

[5] D. Larese and F. Iachello. J. Mol. Struct. 1006, 611-628 (2011).

[6] D. Larese, F. Pérez-Bernal, and F. Iachello. J. Mol. Struct. 1051, 310-327 (2013).

[7] A. Leviatan, M.W. Kirson, Ann. Phys. NY 188, 142 (1988)

[8] F. Iachello and R. D. Levine. Algebraic Theory of Molecules. Oxford University Press, Oxford, 
1995.

[9] F. Iachello and A. Arima. The Interacting Boson Model. Cambridge University Press, Cambridge, 1987.

[10] S.R. Gadre, S.B. Sears, S.J. Chakravorty, and R.D. Bendale, Phys. Rev. A 32 (1985) 2602.

[11] K.Ch. Chatzisavvas, Ch.C. Moustakidis, and C.P. Panos, J. Chem. Phys. 123 (2005) 174111.

[12] R. J. Yánez, W. Van Assche and J. S. Dehesa, Phys. Rev. A 32, 3065 (1994).

[13] M. W. Coffey, J. Phys. A, Math. Gen. 36 (2003) 7441.

[14] E. Romera, P. Sánchez-Morena and J. S. Dehesa, Chem. Phys. Lett. 414468 (2005).

[15] J. B. Szabó, K. D. Sen and Á. Nagy, Phys. Lett. A 3722428 (2008).

[16] J. Sañudo and R. López-Ruiz, Phys. Lett. A 372 (2008) 5283.

[17] R. A. Fisher, Proc. Cambridge Philos. Soc.22, 700 (1925).

[18] C. E. Shannon, Bell Syst. Tech. J. 27, (1948) 379.

[19] C. Vignat and J. F. Bercher, Phys. Lett. A 31227 (2003).

[20] E. Romera and J. S. Dehesa, J. Chem. Phys. 120, 8906 (2004).

[21] J. C. Angulo, J. Antolí and K.D. Sen, Phys. Lett. A 372 (2008) 670.

[22] R. López-Ruiz, H. L. Mancini, X. Calbet, Phys. Lett. A 209 (1995) 321.

[23] R.G. Catalan, J. Garay, R. López-Ruiz, Phys. Rev. E 66 (2002) 011102.

[24] Á. Nagy, K. D. Sen and H. E. Montgomery, Phys. Lett. A 373 (2009) 2552.

[25] E. Romera, M. Calixto and Á. Nagy, Europhys. Lett. 97, 20011 (2012).

[26] M. Calixto, Á. Nagy, I. Paraleda and E. Romera, Phys. Rev. A. 85, 053813 (2012).

[27] E. Romera, R. del Real, M. Calixto, S. Nagy, and Á. Nagy, J. Math. Chem. 51, 620-636 (2013)

[28] E. Romera, R. del Real, M. Calixto, Phys. Rev. A 85, 053831, (2012).

[29] M. Calixto, R. del Real, E. Romera, Phys. Rev A 86, 032508 (2012).

[30] M. Calixto, E. Romera and R. del Real, J. Phys. A 45, 365301 (2012)

[31] Á. Nagy and E. Romera, Physica A 391 (2012) 3650.

[32] R. López-Ruiz, Á. Nagy, E. Romera, J. Sañudo, J. of Math. Phys. 50, 123528 (2009).

[33] J. C. Angulo, J. Antolin and R. O. Esquivel, in Statistical Complexity ed. K. D. Sen, (Springer, Berlin, 2011) pp 167.

[34] E. Romera, R. López-Ruiz, J. Sanudo and Á. Nagy, Int. Rev. Phys. 3 (2009) 207. 